\title{
Perfusion Imaging with SPECT in the Era of Pathophysiology-Based Biomarkers for Alzheimer's Disease
}

\author{
Markus Weih, ${ }^{1}$ Ümüt Degirmenci, ${ }^{1}$ Sebastian Kreil, ${ }^{1}$ Piotr Lewczuk, ${ }^{1}$ Daniela Schmidt, ${ }^{2}$ \\ Johannes Kornhuber, ${ }^{1}$ and Torsten Kuwert ${ }^{2}$ \\ ${ }^{1}$ Department for Psychiatry and Psychiatry, University of Erlangen-Nuermberg, Schwabachanlage 6, 91054 Erlangen, Germany \\ ${ }^{2}$ Clinic of Nuclear Medicine, University of Erlangen-Nuermberg, Schwabachanlage 6, 91054 Erlangen, Germany \\ Correspondence should be addressed to Markus Weih, markus.weih@uk-erlangen.de
}

Received 8 October 2010; Accepted 15 November 2010

Academic Editor: Adam S. Fleisher

Copyright () 2010 Markus Weih et al. This is an open access article distributed under the Creative Commons Attribution License, which permits unrestricted use, distribution, and reproduction in any medium, provided the original work is properly cited.

SPECT allows registration of regional cerebral blood flow (rCBF) which is altered in a characteristic temporoparietal pattern in Alzheimer's Dementia. Numerous studies have shown the diagnostic value of reduced cerebral blood flow and metabolic changes using perfusion SPECT and FDG-PEPT in AD diagnosis as well as in differential diagnosis against frontotemporal dementia, dementia with Lewy bodies and vascular disease. Recently more pathophysiology-based biomarkers in CSF and Amyloid-PET tracers have been developed that probably have a higher diagnostic accuracy than the more indirect rCBF changes seen in perfusion SPECT. In the paper review, we describe recent advances in AD biomarkers as well as improvements in the SPECT technique.

\section{Introduction}

Memory problems in elderly patients are common and can be due to a wide range of conditions. Alzheimer's Disease (AD) accounts for a large amount of cases, especially when gradual onset and further cognitive problems like aphasia, apraxia, or reduced activities of daily life are present. AD originally has been a pathologic diagnosis. The "golden standard" of AD are comprehensive clinical criteria (like NINCDS-ADRDA, DSM-IV, or ICD-10). These criteria have been validated against pathology with a wide range of sensitivity (65\%-100\%) and specificity (65\%-90\%) [1]. Thus, during the last decades, imaging and cerebrospinal fluid (CSF) taps were then performed to rule out secondary memory disorders, for example, due to intracranial mass or infection (like syphilis). As a first approach to in vivo AD imaging, single proton emission computed tomography (SPECT) with perfusion tracers was developed and validated approximately 25 years ago. Perfusion SPECT shows a characteristic (nonvascular) pattern in parietal-temporal cortical areas that indirectly reflect the underlying spatial distribution of neurofibrillary and amyloid pathology. The coupling of fading neural activity and glucose metabolism to reduced regional cerebral blood flow ( $\mathrm{rCBF}$ ) allowed for the first time real albeit indirect "functional imaging" in neurodegenerative diseases using SPECT and later FDG-PET. More recently, advances in CSF protein assays and the development of specific amyloid-binding Positron Emission Tomography (PET) tracers allowed direct and pathophysiology-based tests and imaging. Here we would like to review the diagnostic value, accuracy, advantages, and disadvantages of perfusion SPECT in comparison to these more advanced CSF and PET biomarkers.

\section{Diagnostic Value of Perfusion SPECT}

Since 1991 more than 50 studies have been performed to compare the diagnostic value of SPECT (mostly ${ }^{99 \mathrm{~m}} \mathrm{Tc}$ $\mathrm{HMPAO}$ ) in $\mathrm{AD}$ versus normal volunteers, patients without neurodegenerative dementia (like depression) or other forms of dementia like frontotemporal dementia (FTD), dementia with Lewy bodies (DLB), or vascular dementia [2]. Taken together these studies clearly showed added value of SPECT for or against a clinical diagnosis of AD. A comprehensive review of Dougall et al. [3] identified 389 publications on 
this issue. After exclusion of nonrelevant studies using a methodology checklist the authors found 37 studies of clinical AD versus normal controls (comprising 1559 subjects) and 13 studies of $\mathrm{AD}$ versus nondemented control patients (1082 patients). The case-control studies with $\mathrm{AD}$ patients versus normal subjects were too heterogeneous to compare. The studies of clinical AD versus nondemented patients showed pooled sensitivities/specificities of $66 \%$ and $79 \%$, respectively, yielding a diagnostic odds ratio of 8.2 [3]. In our own data base, we found sensitivities of 48\%-60\% [4] and a specificity of $90 \%$ [5]. Comparison of blinded SPECT versus a pathological confirmed diagnosis of dementia yielded SPECT sensitivity/specificity of $63 \%-86 \%$ and $73 \%-93 \%$ $[6,7]$. Another important clinical question is the value of perfusion SPECT to predict progression from mild cognitive impairment (MCI) to AD. Devanand et al. [8] found a sensitivity of only $42 \%$ but a specificity of $82 \%$ for perfusion SPECT. Taken together these results suggest that SPECT has a sensitivity for AD that is most likely below the desired threshold of a valid biomarker, but a high specificity, making SPECT at least a useful diagnostic tool to rule out AD. In Table 1 the individual components of perfusion SPECT are displayed against other techniques.

\section{Comparison of Perfusion SPECT and FDG-PET}

The standard radionuclide in clinical PET centers is FDG $\left({ }^{18} \mathrm{~F}\right.$ tagged to Deoxyglucose). FDG has long been the traditional method to measure secondary glucose metabolism changes downstream the neurodegenerative process in AD. Since cerebral metabolism and blood flow are coupled, there is a similar pattern of regional disturbed glucose metabolic rates (rCMRGlu) in the temporal-parietal cortex, less in the prefrontal area as mentioned before. The first description appeared in 1982 by Farkas et al. [9]. Since then, many other groups confirmed the diagnostic value of FGG-PET in AD. In a meta-analysis of 15 articles, Patwardhan et al. found a pooled sensitivity of $86 \%$ and a specificity of $86 \%$ [10]. FDGPET and perfusion SPECT are the two most prominently available functional imaging methods available to clinicians worldwide.

Direct comparison of both techniques in the same population has not been performed to our knowledge. When comparing the diagnostic accuracy based on meta-analytic data, there is some evidence for a higher sensitivity and specificity of FDG-PET when compared to perfusion SPECT.

\section{Diagnostic Value of CSF-Based Biomarkers}

Since the identification of Amyloid Precursor Protein (APP) in $\mathrm{AD}$ pathophysiology, there have been efforts to find appropriate APP-based CSF tests. However, initial assays measured all amyloid peptides and were not specific enough. After the pioneering work of Motter et al. in 1995 [11] who found a specific reduction of beta-amyloid peptide-42 in CSF, these results were then confirmed by many other groups. On average there is a reduction of beta-amyloid peptide- 42 of approximately $40 \%-50 \%$, and the tests have a sensitivity and specificity of approximately $85 \%$. Other publications evaluated whether tau-pathology is also reflected in CSF changes. CSF levels of the microtubule-associated tau protein are typically increased (on average 2.5-fold) in $\mathrm{AD}$ and reflect neuronal damage [12]. Data from more than 35 single-center studies comprising more than $2500 \mathrm{AD}$ patients and 1400 controls, meta-analyses, and consensus papers [13] are now available. When the specificity is set to $90 \%$ (which is a desirable value for biomarkers) total-tau has a sensitivity of app. $80 \%$ [14].

Hyperphosphorylation of tau (at position Threonine 181, 231 and Serine 199) occurs solely in $\mathrm{AD}$ and is therefore theoretically a more specific biomarker. However, studies showed large differences in sensitivity and specificity. A meta-analysis of 51 single-center studies by Mitchell [15] showed a pooled sensitivity of $78 \%$ and a specificity of $88 \%$ against patients without cognitive impairment. More recently, the different CSF markers have been combined [16] and the interpretation algorithms have been refined [17]. When evaluated in larger multicenter trials in patients with $\mathrm{MCI}, \mathrm{AD}$ and nondemented patients or healthy subjects, the sensitivity of the combined CSF biomarkers (Amyloid peptide-42- and tau markers) to detect AD in MCI patients was then $85 \%-95 \%$ and the specificity $72 \%-83 \%[18,19]$. Other biomarkers like soluble APP are under investigation [20] and need to be validated. As a disadvantage it turned out in the CSF multicenter trials that there are larger than expected interlaboratory discrepancies [21].

\section{Amyloid- and Tau-PET}

The Pittsburgh Compound-B $\left({ }^{11} \mathrm{C}-\mathrm{PiB}\right)$ was the first of a new family of PET tracers that have shown to be of high diagnostic value in $\mathrm{AD}$. Specific binding of $\mathrm{PiB}$ to brain Amyloid has been extensively studied in case-control studies and longitudinal observations since 2004 [22]. The area under the curve (AUC) in receiver operator characteristics (ROCs) for $\mathrm{PiB}$ is around 0.8-0.9 and can be improved to 0.94 when reserve variables like education, brain volume, gender, physical health, and medications are taken into account [23]. The main limitation for ${ }^{11} \mathrm{C}$-based PET tracers is their short half-life (appr. 20 minutes) which restricts their use to dedicated research sites with on-site cyclotrons and radiochemistry labs. ${ }^{18} \mathrm{~F}$-based tracers have a longer half-life (appr. 110 minutes). The first ${ }^{18} \mathrm{~F}$ tracer for $\mathrm{AD}$ was FDDNP and developed to recognize both plaques and tangles. This could overcome the problem that some patients have extensive plaque formation but no dementia. The AUC for global rating of the FDDNP-PET could be up to 1.0 for $\mathrm{AD}$ versus control, 0.95 for $\mathrm{MCI}$ versus control and 0.98 for AD versus MCI. These promising results suggest that Amyloid-PETs or combined Amyloid-Tau-PETs may have a diagnostic value superior to other imaging tools like FDG-PET or volumetric MRI [24] in prevalent AD. Also, these tracers have been studies in MCI as a tool for early diagnosis but the results need to be validated in independent studies. Other studies with ${ }^{18} \mathrm{~F}$-PET markers 
TABle 1: Perfusion SPECT versus biomarkers in AD.

\begin{tabular}{|c|c|c|c|c|}
\hline Method & FDG-PET & Perfusion-SPECT & CSF & Amyloid-PET \\
\hline First description & Farkas et al., 1982 [9] & Launes et al., 1991 [2] & $\begin{array}{l}\text { Motter et al., } 1995 \\
\text { [32] }\end{array}$ & Klunk et al., 2004 [22] \\
\hline Diagnostic accuracy & {$[10]$} & {$[3]$} & {$[13,15]$} & {$[23]$} \\
\hline Sensitivity & $86 \%$ (CI: 76\%-93\%) & $66 \%$ (CI: 62\%-69\%) & $80 \%-85 \%$ & appr. $85 \%-100 \% \%$ \\
\hline Specificity & $86 \%$ (CI: 72\%-93\%) & 79\% (CI: 75\%-83\%) & $85 \%-90 \%$ & appr. $85 \%-100 \%$ \\
\hline Meta-analysis & Yes & Yes & Yes & No \\
\hline Clinical availability & Academic centers & Greater hospitals & $\begin{array}{l}\text { Theoretically } \\
\text { everywhere }\end{array}$ & Academic centers \\
\hline Equipment & Cycloton & Gamma-camera & ELISA reader & Cycloton \\
\hline Cost Estimation & $>1000$ EUR & $<300$ EUR & $<200$ EUR & >2000 EUR \\
\hline $\begin{array}{l}\text { Expertise for } \\
\text { result interpretation }\end{array}$ & High & High & High & High \\
\hline \multirow{3}{*}{$\begin{array}{l}\text { Treatment options if test } \\
\text { positive }\end{array}$} & MCI: negative & MCI: negative & MCI: negative & MCI: negative \\
\hline & AD: positive & AD: positive & AD: positive & AD: positive \\
\hline & & $\begin{array}{l}\text { DLB: off-label } \\
\text { FTD: negative }\end{array}$ & & \\
\hline
\end{tabular}

CI: 95\% confidence interval. MCI: mild cognitive impairment.

like Flutemetamol $\left(\mathrm{PiB}\right.$ with $\left.{ }^{18} \mathrm{~F}\right)$ are in phase II clinical studies [25]. Florbetapir (18F-AV-45) is the most widely studied ${ }^{18} \mathrm{~F}$ Amyloid imaging agent [26] and could receive FDA approval within next time. Other compounds like Florbetaben (ClinicalTrial NCT01020838) are in phase III clinical studies.

\section{Differential Diagnosis: Vascular and Frontotemporal Dementia, Dementia with Lewy-Bodies}

Although AD is the most prevalent dementia, several other neurodegenerative disorders have to be taken into account by the clinician. Vascular dementia is usually characterized by a past medical history with the presence of typical cardiovascular risk factors, stepwise deterioration, and vascular lesions on MRI or CT. Still, Perfusion SPECT has been used to discriminate vascular from primary neurodegenerative dementia. According to the meta-analysis of Dougall et al., the pooled weighted sensitivity and specificity against $\mathrm{AD}$ is $71 \%$, respectively $76 \%[3]$.

Using clinical features alone, the differential diagnosis of frontotemporal dementia (FTD) and Dementia with LewyBodies (DLB) is sometimes difficult. FTD is a heterogeneous disease and in contrast to $\mathrm{AD}$ no established specific and validated biomarkers are available. In the above-mentioned review of Dougall et al. 2004 [3], the authors found a pooled sensitivity and specificity of SPECT for discriminating AD from FTD of $72 \%$ and $78 \%$, respectively The diagnostic odds ratio was 8.4 and is in the same range as that for $\mathrm{AD}$ versus control. In pathologically confirmed FTD cases, McNeill et al. found a sensitivity of bilateral frontal lobe CBF reduction of $80 \%$ and a specificity of $81 \%$ versus $\mathrm{AD}$ [27]. In a larger sample of FTD patients, Mendez et al. found a sensitivity/specificity of $91 \%$ and $75 \%$ and a negative predictive value of $90 \%$ [28]. These results suggest that SPECT provides useful additional information in discriminating $\mathrm{AD}$ from FTD. Some patients with Dementia with Lewy bodies (DLB) have prominent cognitive deficits but MRI usually does not show the characteristic global atrophy as seen in AD. Here several nuclear medicine techniques may be of advantage like assessment of occipital hypoperfusion alone [29] or in combination with MRI [30]. If symptoms of Parkinson's disease are predominant in DLB, Iodine-123 FP-CIT SPECT is of diagnostic value [31]. The characteristic sympathetic denervation in DLB can be assessed using Iodine-123MIBG in a very specific manner [29]. Given the fact that SPECT scanners are available in many larger hospitals, the differential diagnosis of FTD and DLB against AD with SPECT is feasible and clinicaly relevant, since antidementive drugs like cholinesterase-inhibitors are unlikely to be of therapeutic benefit in FTD, but are approved for $\mathrm{AD}$, Parkinson's disease dementia (PDD) and are beneficial in DLB (positive therapeutic studies, but off-label use).

\section{Technical Improvements}

The image acquisition technique in SPECT is prone to signal degradation due to different physical phenomena like depthdependent blurring, photon attenuation and scattering.

SPECT images are evaluated similar to other imaging techniques like MRI or CT, inferring bias due to subjective image interpretation. Technical limitations in the past made iterative image reconstruction necessary [33]. In recent years, considerable advances have been made in this area like the introduction of hybrid SPECT/CT devices (for simultaneous registration of anatomy and function) and optimized, fast imaging reconstruction hard- and software. These advances now allow improved calibration and quantification of SPECT images. The coregistration of SPECT images with structural imaging and advances in automated anatomical labeling (AAL) opened the possibility to identify voxels of interest 
(VOI) which increases the originally limited spatial resolution of SPECT ultimately leading to better discrimination of AD patients. In addition, Pagani et al. [34] as well as Merhof et al. [35] has recently shown that rCBF patterns together with anatomical information can be subjected to principal component analysis (PCA) resp. multivariate analysis, which allows to draw further conclusion about disturbed functional connectivity between brain regions in dementia. Perfusion imaging using arterial spin labeling MRI is a standard technique in cerebrovascular disease and has been applied to $\mathrm{AD}$ diagnosis and differential diagnosis to FTD with promising results $[36,37]$ but the exact diagnostic value parameters like sensitivity, specificity have not yet been delineated in detail.

\section{Areas of Uncertainty}

It is still unclear whether the "AD pattern" in perfusion SPECT merely reflects cortical neurodegeneration or the secondary cholinergic deficit following degeneration of the basal nucleus of Meynert. Studies that both investigated CSF biomarkers and SPECT showed no correlation $[5,38]$. Simultaneous registration of the cholinergic deficit (e.g., using Nicotinic 123I-5IA-85380 SPECT [39]) and perfusion SPECT or Amyloid-PET with perfusion SPECT could help to resolve this issue.

In perfusion SPECT usually lobar hypoperfusion is registrated. More recently, MRI techniques have shown that there is also compensatory hyperperfusion in AD and FTD [36]. Possible underlying mechanisms are partial deafferentation or a cognitive reserve mechanism. It would be interesting to confirm these results in perfusion SPECT, resp. to explore the diagnostic value of hyperperfusion in correlation to reserve variables.

\section{Conclusions}

Perfusion SPECT and FDG-PET are metabolic based and were the first methods of "functional" brain imaging in Alzheimer's disease showing hypoperfusion in the temporoparietal regions with the highest load with plaques and tangles in postmortem brains with AD.

This has lead to a widespread use of perfusion SPECT and FDG-PET as a diagnostic tool in AD. Since specificity exceeds sensitivity the diagnostic value of perfusion SPECT to rule out $\mathrm{AD}$ usually is higher than to confirm it. However, accuracy of any diagnostic tool is critically dependent on disease severity and the population under investigation. In addition, perfusion SPECT is useful in discriminating vascular dementia, FTD, and DLB in the absence of validated specific biomarkers for these conditions. More recently pathophysiology-based CSF-biomarkers, especially beta-amyloid peptide- 42 and tau protein, have been investigated with a diagnostic accuracy that seems to be superior to perfusion SPECT and FDG-PET. In addition, due to their direct reflection of the underlying process, CSF biomarkers (alone or in combination) are also suited for early diagnosis, for example, in patients with MCI, where the diagnostic value for SPECT is even lower than in prevalent AD. In contrast to CSF assays, PET biomarkers have the advantage of showing the anatomic distribution of pathology and were first available for amyloid plaques $\left({ }^{11} \mathrm{C} \mathrm{PiB}\right)$. Now, there is development of longer-lasting ${ }^{18} \mathrm{~F}$ PET tracers as well first evidence that plaques and tangles can be imaged with a single PET compound, but the results have to be confirmed in independent studies.

\section{References}

[1] D. S. Knopman, S. T. DeKosky, J. L. Cummings et al., "Practice parameter: diagnosis of dementia (an evidence-based review): report of the quality standards subcommittee of the american academy of neurology," Neurology, vol. 56, no. 9, pp. 11431153, 2001.

[2] J. Launes, R. Sulkava, T. Erkinjuntti, P. Nikkinen, L. Lindroth, and K. M. Liewendahl Iivanainen, "99Tcm-HMPAO SPECT in suspected dementia," Nuclear Medicine Communications, vol. 12, no. 9, pp. 757-765, 1991.

[3] N. J. Dougall, S. Bruggink, and K. P. Ebmeier, "Systematic review of the diagnostic accuracy of ${ }^{99 m} \mathrm{Tc}-\mathrm{HMPAO}$ - SPECT in dementia," American Journal of Geriatric Psychiatry, vol. 12, no. 6, pp. 554-570, 2004.

[4] M. Weih, M. Krinninger, R. Zimmermann et al., "Vergleichende sensitivität der neurochemischen demenzdiagnostik im liquor und $\mathrm{rCBF}$ im ${ }^{99 m} \mathrm{Tc}$-SPECT bei patienten mit Alzheimerdemenz," Fortschritte der Neurologie-Psychiatrie, vol. 77, no. 7, pp. 407-411, 2009.

[5] D. Schmidt, R. Zimmermann, P. Lewczuk et al., "Confirmation rate of blinded ${ }^{99 m}$ Tc-SPECT compared to neurochemical dementia biomarkers in CSF in patients with Alzheimer disease," Journal of Neural Transmission, vol. 117, no. 9, pp. 1111-1114, 2010.

[6] F. J. Bonte, M. F. Weiner, E. H. Bigio, and C. L. White, "Brain blood flow in the dementias: SPECT with histopathologic correlation in 54 patients," Radiology, vol. 202, no. 3, pp. 793797, 1997.

[7] W. Jagust, R. Thisted, M. D. Devous et al., "SPECT perfusion imaging in the diagnosis of alzheimer's disease: a clinicalpathologic study," Neurology, vol. 56, no. 7, pp. 950-956, 2001.

[8] D. P. Devanand, R. L. van Heertum, L. S. Kegeles et al., “ ${ }^{99 m}$ Tc hexamethyl-propylene-aminoxime single-photon emission computed tomography prediction of conversion from mild cognitive impairment to Alzheimer disease," American Journal of Geriatric Psychiatry, vol. 18, no. 11, pp. 959-972, 2010.

[9] T. Farkas, S. H. Ferris, and A. P. Wolf, "F-2-Deoxy-2-fluoro$\mathrm{D}$-glucose as a tracer in the positron emission tomographic study of senile dementia," American Journal of Psychiatry, vol. 139, no. 3, pp. 352-353, 1982.

[10] M. B. Patwardhan, D. C. McCrory, D. B. Matchar, G. P. Samsa, and O. T. Rutschmann, "Alzheimer disease: operating characteristics of PET-a meta-analysis," Radiology, vol. 231, no. 1, pp. 73-80, 2004.

[11] R. Motter, C. Vigo-Pelfrey, D. Kholodenko et al., "Reduction of $\beta$-amyloid peptide in the cerebrospinal fluid of patients with Alzheimer's disease," Annals of Neurology, vol. 38, no. 4, pp. 643-648, 1995.

[12] P. Lewczuk, H. Esselmann, M. Bibl et al., “Tau protein phosphorylated at threonine 181 in CSF as a neurochemical 
biomarker in Alzheimer's disease: original data and review of the literature," Journal of Molecular Neuroscience, vol. 23, no. 1-2, pp. 115-122, 2004.

[13] J. Wiltfang, P. Lewczuk, P. Riederer et al., "Consensus Paper of the WFSBP Task Force on Biological Markers of Dementia: the role of CSF and blood analysis in the early and differential diagnosis of dementia," World Journal of Biological Psychiatry, vol. 6, no. 2, pp. 69-84, 2005.

[14] K. Blennow and H. Hampel, "CSF markers for incipient Alzheimer's disease," Lancet Neurology, vol. 2, no. 10, pp. 605613, 2003.

[15] A. J. Mitchell, "CSF phosphorylated tau in the diagnosis and prognosis of mild cognitive impairment and Alzheimer's disease: a meta-analysis of 51 studies," Journal of Neurology, Neurosurgery and Psychiatry, vol. 80, no. 9, pp. 966-975, 2009.

[16] P. Lewczuk, H. Esselmann, M. Otto et al., "Neurochemical diagnosis of Alzheimer's dementia by CSF A $\beta 42, \mathrm{~A} \beta 42 / \mathrm{A} \beta 40$ ratio and total tau," Neurobiology of Aging, vol. 25, no. 3, pp. 273-281, 2004.

[17] P. Lewczuk, R. Zimmermann, J. Wiltfang, and J. Kornhuber, "Neurochemical dementia diagnostics: a simple algorithm for interpretation of the CSF biomarkers," Journal of Neural Transmission, vol. 116, no. 9, pp. 1163-1167, 2009.

[18] N. Mattsson, H. Zetterberg, O. Hansson et al., "CSF biomarkers and incipient Alzheimer disease in patients with mild cognitive impairment," Journal of the American Medical Association, vol. 302, no. 4, pp. 385-393, 2009.

[19] O. Hansson, H. Zetterberg, P. Buchhave, E. Londos, K. Blennow, and L. Minthon, "Association between CSF biomarkers and incipient Alzheimer's disease in patients with mild cognitive impairment: a follow-up study," Lancet Neurology, vol. 5, no. 3, pp. 228-234, 2006.

[20] P. Lewczuk, H. Kamrowski-Kruck, O. Peters et al., "Soluble amyloid precursor proteins in the cerebrospinal fluid as novel potential biomarkers of Alzheimer's disease: a multicenter study," Molecular Psychiatry, vol. 15, no. 2, pp. 138-145, 2010.

[21] N. Mattsson, H. Zetterberg, O. Hansson et al., "CSF biomarkers and incipient Alzheimer disease in patients with mild cognitive impairment," Journal of the American Medical Association, vol. 302, no. 4, pp. 385-393, 2009.

[22] W. E. Klunk, H. Engler, A. Nordberg et al., "Imaging brain amyloid in Alzheimer's disease with Pittsburgh compound-B," Annals of Neurology, vol. 55, no. 3, pp. 306-319, 2004.

[23] C. M. Roe, M. A. Mintun, N. Ghoshal et al., "Alzheimer disease identification using amyloid imaging and reserve variables: proof of concept," Neurology, vol. 75, no. 1, pp. 42-48, 2010.

[24] G. W. Small, V. Kepe, L. M. Ercoli et al., "PET of brain amyloid and tau in mild cognitive impairment," New England Journal of Medicine, vol. 355, no. 25, pp. 2652-2663, 2006.

[25] R. Vandenberghe, K. van Laere, A. Ivanoiu et al., "18Fflutemetamol amyloid imaging in Alzheimer disease and mild cognitive impairment a phase 2 trial," Annals of Neurology, vol. 68, no. 3, pp. 319-329, 2010.

[26] E. R. L. C. Vardy, T. Langheinrich, R. Hinz et al., "POD14 Amyloid PET using 18F-AV-45 in Alzheimer's disease and frontotemporal dementia: first UK results " Journal of $\mathrm{Neu}$ rology, Neurosurgery \& Psychiatry, vol. 81, no. 11, article e45, 2010.

[27] R. McNeill, G. M. Sare, M. Manoharan et al., "Accuracy of single-photon emission computed tomography in differentiating frontotemporal dementia from Alzheimer's disease," Journal of Neurology, Neurosurgery and Psychiatry, vol. 78, no. 4, pp. 350-355, 2007.
[28] M. F. Mendez, J. S. Shapira, A. McMurtray, E. Licht, and B. L. Miller, "Accuracy of the clinical evaluation for frontotemporal dementia," Archives of Neurology, vol. 64, no. 6, pp. 830-835, 2007.

[29] S. Shimizu, H. Hanyu, H. Kanetaka, T. Iwamoto, K. Koizumi, and K. Abe, "Differentiation of dementia with lewy bodies from alzheimer's disease using brain SPECT," Dementia and Geriatric Cognitive Disorders, vol. 20, no. 1, pp. 25-30, 2005.

[30] H. Goto, K. Ishii, T. Uemura et al., "Differential diagnosis of dementia with lewy bodies and alzheimer disease using combined MR imaging and brain perfusion single-photon emission tomography," American Journal of Neuroradiology, vol. 31, no. 4, pp. 720-725, 2010.

[31] G. Tissingh, J. Booij, P. Bergmans et al., "Iodine-123-N- $\omega-$ fluoropropyl-2/ $\beta$-carbomethoxy $3 \beta$-(4-iodophenyl)tropane SPECT in healthy controls and early-stage, drug-naive Parkinson's disease," Journal of Nuclear Medicine, vol. 39, no. 7, pp. 1143-1148, 1998.

[32] R. Motter, C. Vigo-Pelfrey, D. Kholodenko et al., "Reduction of $\beta$-amyloid peptide in the cerebrospinal fluid of patients with Alzheimer's disease," Annals of Neurology, vol. 38, no. 4, pp. 643-648, 1995.

[33] J. Zeintl, A. H. Vija, A. Yahil, J. Hornegger, and T. Kuwert, "Quantitative accuracy of clinical ${ }^{99 m} \mathrm{Tc}$ SPECT/CT using ordered-subset expectation maximization with 3-dimensional resolution recovery, attenuation, and scatter correction," Journal of Nuclear Medicine, vol. 51, no. 6, pp. 921-928, 2010.

[34] M. Pagani, D. Salmaso, G. Rodriguez, D. Nardo, and F. Nobili, "Principal component analysis in mild and moderate Alzheimer's disease - a novel approach to clinical diagnosis," Psychiatry Research, vol. 173, no. 1, pp. 8-14, 2009.

[35] D. Merhof, P. J. Markiewicz, G. Platsch et al., "Optimized data preprocessing for multivariate analysis applied to Tc-ECD SPECT data sets of Alzheimer's patients and asymptomatic controls," Journal of Cerebral Blood Flow and Metabolism. In press.

[36] W. T. Hu, Z. Wang, V. M.-Y. Lee, J. Q. Trojanowski, J. A. Detre, and M. Grossman, "Distinct cerebral perfusion patterns in FTLD and AD,” Neurology, vol. 75, no. 10, pp. 881-888, 2010.

[37] C. Luckhaus, M. Cohnen, M. O. Flüss et al., "The relation of regional cerebral perfusion and atrophy in mild cognitive impairment (MCI) and early Alzheimer's dementia," Psychiatry Research, vol. 183, no. 1, pp. 44-51, 2010.

[38] M. Tsolaki, V. Sakka, G. Gerasimou et al., "Correlation of rCBF (SPECT), CSF tau, and cognitive function in patients with dementia of the Alzheimer's type, other types of dementia, and control subjects," American Journal of Alzheimer's Disease, vol. 16, no. 1, pp. 21-31, 2001.

[39] S. J. Colloby, E. K. Perry, S. Pakrasi et al., "Nicotinic 123I5IA-85380 single photon emission computed tomography as a predictor of cognitive progression in alzheimer's disease and dementia with lewy Bodies," American Journal of Geriatric Psychiatry, vol. 18, no. 1, pp. 86-90, 2010. 


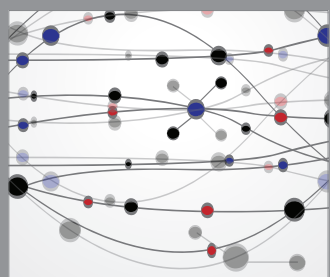

The Scientific World Journal
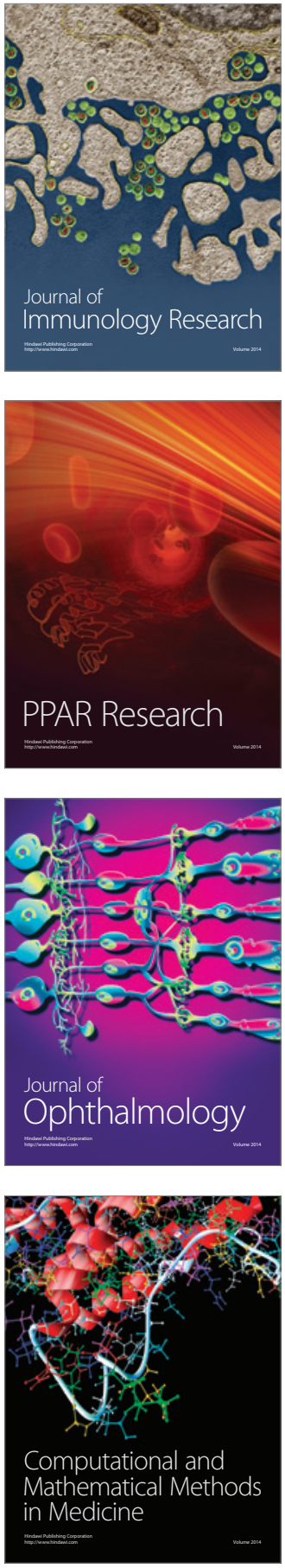

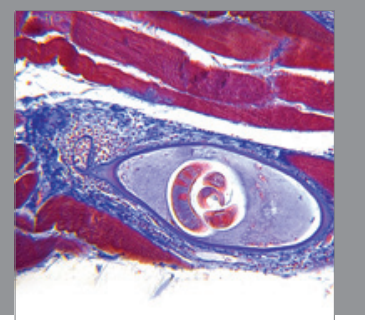

Gastroenterology

Research and Practice
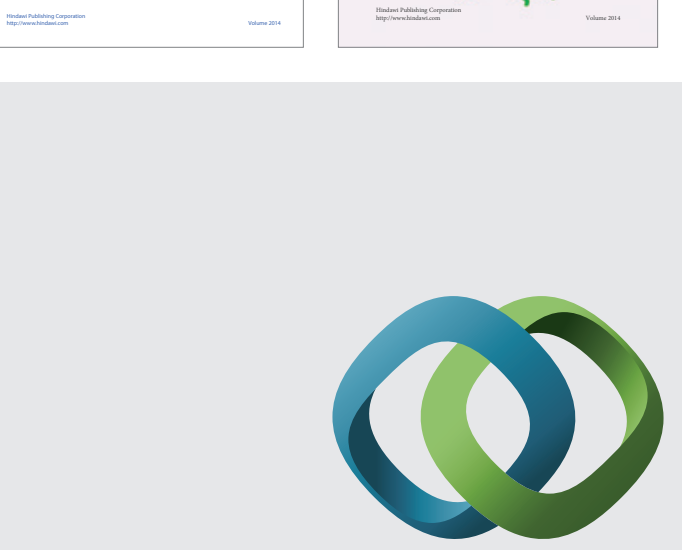

\section{Hindawi}

Submit your manuscripts at

http://www.hindawi.com
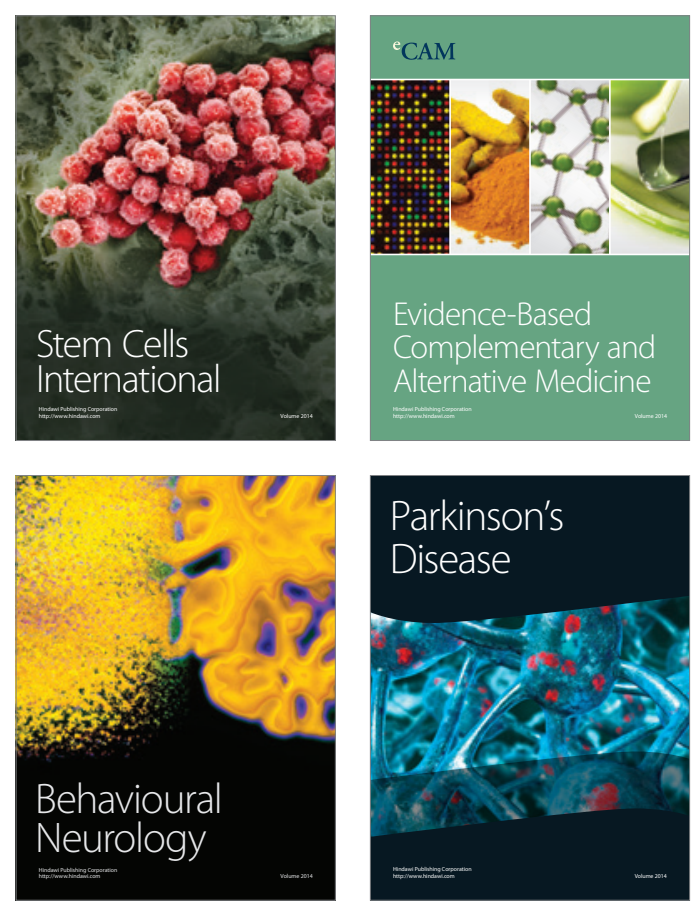

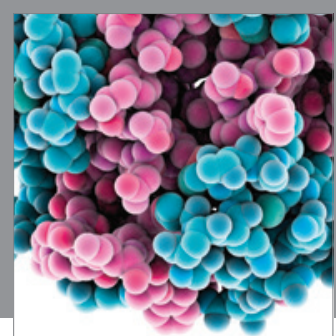

Journal of
Diabetes Research

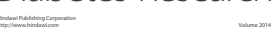

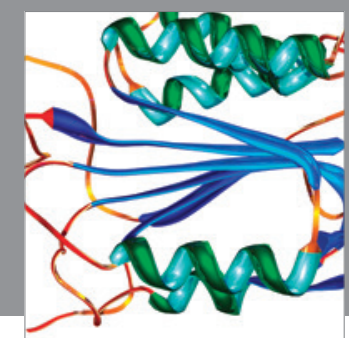

Disease Markers
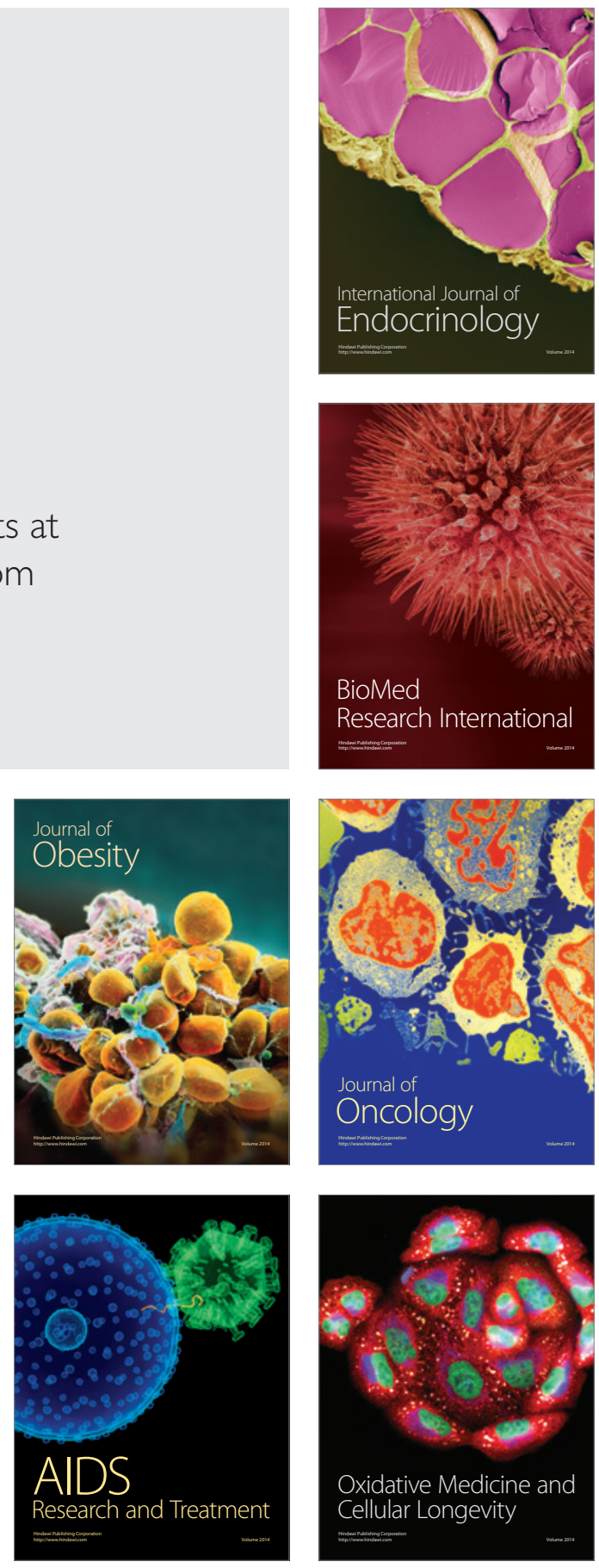